\title{
Measuring the lifecycle of baryonic matter in the Large Magellanic Cloud with the Spitzer SAGE-LMC survey
}

\author{
Margaret Meixner ${ }^{1}$, Jean-Philippe Bernard ${ }^{2}$, Robert D. Blum ${ }^{3}$, \\ Remy Indebetouw ${ }^{4}$, William Reach ${ }^{5}$, Sundar Srinivasan ${ }^{6}$, \\ Marta Sewilo ${ }^{1}$ and Barbara A. Whitney ${ }^{7}$ \\ ${ }^{1}$ Space Telescope Science Institute, 3700 San Martin Dr., Baltimore, MD 21093, USA \\ email: meixner@stsci.edu,mmsewilo@stsci.edu
}

\footnotetext{
${ }^{2}$ Direction de la Recherche, Centre dEtude Spatiale des Rayonnements, 18Avenue Edouard Belin, Toulouse, Cedex F-31055, France email: jean-philippe.bernard@cesr.fr

${ }^{3}$ NOAO, PO Box 26732, Tucson AZ 85726-6732, USA email: rblum@noao.edu

${ }^{4}$ Depart. of Astronomy, University of Virginia, PO Box 3818, Charlottesville, VA 22903, USA email: remy@virginia.edu

${ }^{5}$ Spitzer Science Center, California Institute of Technology, 220-6, Pasadena, CA, 91125, USA email: reach@ipac.caltech.edu

${ }^{6}$ Department of Physics and Astronomy, The Johns Hopkins University, 3400 North Charles St., Baltimore, MD 21218, USA email: sundar@pha.jhu.edu

${ }^{7}$ Space Science Institute, 4750 Walnut St. Suite 205, Boulder, CO 80301, USA email: bwhitney@spacescience.org
}

\begin{abstract}
The recycling of matter between the interstellar medium (ISM) and stars are key evolutionary drivers of a galaxy's baryonic matter. The Spitzer wavelengths provide a sensitive probe of circumstellar and interstellar dust and hence, allow us to study the physical processes of the ISM, the formation of new stars and the injection of mass by evolved stars and their relationships on the galaxy-wide scale of the LMC. Due to its proximity, favorable viewing angle, multi-wavelength information, and measured tidal interactions with the Small Magellanic Cloud (SMC), the LMC is uniquely suited for surveying the agents of a galaxy's evolution (SAGE), the ISM and stars. The SAGE-LMC project is measuring these key transition points in the life cycle of baryonic matter in the LMC. Here we present a connective view of the preliminary quantities estimated from SAGE-LMC for the total mass of the ISM, the galaxy wide star formation rate and the current stellar mass loss return. For context, we compare these numbers to the LMC's stellar mass.
\end{abstract}

Keywords. surveys, stars: AGB and post-AGB, circumstellar matter, stars: formation, stars: mass loss, dust, extinction, ISM: evolution, galaxies: evolution, galaxies: individual (LMC), Magellanic Clouds

\section{The SAGE-LMC survey}

We have performed a uniform and unbiased imaging survey of the Large Magellanic Cloud $\left(\mathrm{LMC}, \sim 7^{\circ} \times 7^{\circ}\right)$, using the $\operatorname{IRAC}(3.6,4.5,5.8$ and $8 \mu \mathrm{m})$ and $\operatorname{MIPS}(24,70$, and $160 \mu \mathrm{m}$ ) instruments on board the Spitzer Space Telescope (Spitzer) in order to survey the agents of a galaxy's evolution (SAGE), through the interaction between the interstellar medium (ISM) and stars in the LMC. Three key science goals determined the 
coverage and depth of the survey. The detection of diffuse ISM with column densities $>1.2 \times 10^{21} \mathrm{H} \mathrm{cm}^{-2}$ permits detailed studies of dust processes in the ISM. SAGE's point source sensitivity enables a complete census of newly formed stars with masses $>3 \mathrm{M}_{\odot}$ that will determine the current star formation rate in the LMC. SAGE's detection of evolved stars with mass-loss rates $>1 \times 10^{-8} \mathrm{M}_{\odot} \mathrm{yr}^{-1}$ will quantify the rate at which evolved stars inject mass into the ISM of the LMC. The observing strategy includes two epochs in 2005, separated by three months, that both mitigate instrumental artifacts and constrain source variability. The science drivers, detailed observing strategy, data processing steps and preliminary results of the SAGE survey are described by Meixner et al. (2006).

The SAGE data are non-proprietary and point source lists are released to the community. The most recent release included an improved epoch 1 catalog, an epoch 2 catalog, of IRAC ( $\sim 4$ million point sources) and MIPS 24 ( 40,000 point sources) photometry, and MIPS 24, 70 and 160 micron images (See poster contribution by Meixner and SAGE-LMC team). Further investigations have followed on the SAGE theme such as SAGE-SMC, a Small Magellanic Cloud survey with Spitzer led by Gordon (see poster contribution), SAGE-Spec, a Spitzer IRS spectroscopic survey of the LMC led by Marwick-Kemper, and HERITAGE, a HERschel Inventory of The Agents of Galaxy Evolution in the Magellanic Clouds led by Meixner (see poster contribution). To learn more about all of these SAGE related projects, visit http://sage.stsci.edu. In order to avoid confusion amongst this expansion of SAGE projects, we have retitled ours SAGE-LMC. Several presentations at this conference have been based on the SAGE-LMC data, the SAGE-LMC team contributions are noted throughout this article. In this contribution, we provide an overview of the mass inventory of the ISM, galaxy wide star formation rate, mass-loss rate return of the asymptotic giant branch (AGB) stars as derived from the SAGE-LMC project.

\section{ISM mass}

A global view of the ISM dust properties derived from the SAGE-LMC data is presented by Bernard et al. (2008) (see also poster contribution by Meixner et al.). Among the results is a derivation of the total mass of the ISM (see Table 1), which appears to be a factor of two larger than currently known from gas measurements of $\mathrm{H} \mathrm{I}(21 \mathrm{~cm}$; Kim et al. 2003) and of CO (Fukui et al. 1999). The MIPS $160 \mu \mathrm{m}$ imaging provides us with the most sensitive measures of the total ISM mass in galaxies. Because the optical depth is quite low at $160 \mu \mathrm{m}$, its emission is sensitive to all of the dust and, by inference the gas, in a galaxy independent of the physical conditions of the gas which can cause gas tracers to be only selective measures of the ISM column density.

Bernard et al. (2008) created an infrared excess image (Fig. 1) that is derived as follows. The dust optical depth map at $160 \mu \mathrm{m}$ is scaled to a total gas column density map by multiplying it by a gas-to-dust mass ratio determined from the lowest optical depth regions of the map. The ISM mass listed in Table 1 is integrated over the total area of this map. From this total gas column density map, we subtract the gas column density maps derived from the $\mathrm{HI}$ and $\mathrm{CO}$ maps. The result is the infrared excess map shown in Fig. 1. If the $\mathrm{H}$ I and CO maps had detected all of the ISM mass, as measured by the MIPS $160 \mu \mathrm{m}$ map, then Fig. 1 would be blank. However, it is not blank and instead appears very similar in distribution to the $\mathrm{H}$ I image. This infrared excess map may be tracing the location of $\mathrm{H}_{2}$ gas mass not detected by $\mathrm{CO}$, or cold $\mathrm{H}$ I not detected by the 21 $\mathrm{cm}$ line emission. On the other extreme, it may be tracing (rather large) variations in the dust-to-gas mass ratio, although Bernard et al. (2008) tend to think the dust extinction 

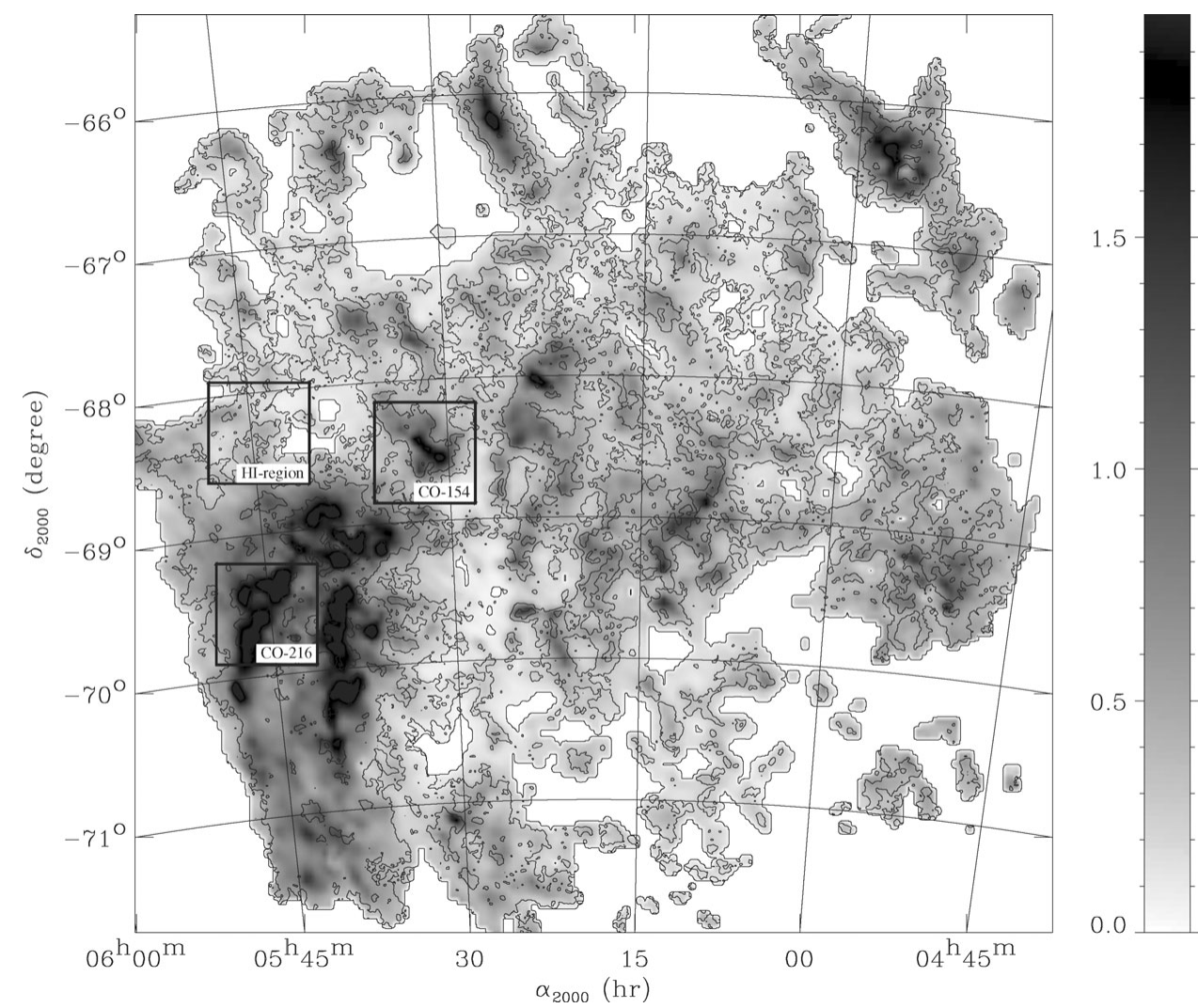

Figure 1. Map of $N_{H}^{X}$ in units of $10^{22} \mathrm{H} / \mathrm{cm}^{2}$. White regions were removed based on their high relative errors. The contours show the distribution of the $\mathrm{H} \mathrm{I}$ emission of Kim et al. (2003). From Bernard et al. (2008).

maps do not support this interpretation. Additional ISM results based on the SAGELMC data are presented in these proceedings by Marble et al. (variations in Aromatic features in the ISM).

\section{Star formation rate}

An overview of the Star Formation as seen with the SAGE-LMC survey is presented by Whitney et al. (2008). The discovery of 1000 new candidate young stellar objects (YSOs) and our preliminary characterization of them, described in this proceedings by Indebetouw, demarks a major step forward in our understanding of star formation processes in the LMC. However, the list is just a first attempt and collects mainly the dustiest, and most massive sources. One of the properties determined from the model fits to the YSOs is the mass. Figure 2 shows the mass distribution of the published YSO candidates which reside over the whole LMC. Whitney et al. (2008) fitted a Kroupa (2001) initial mass function to the part of the mass distribution in which they had the most confidence, the more massive star end. They derive a star formation rate of $0.1 \mathrm{M}_{\odot} \mathrm{yr}^{-1}$ (see Table 1). This bottoms up approach to star formation rate provides a comparable value to that measured from the $\mathrm{H} \alpha$ and far-infrared continuum emission. However, this measurement is almost certainly a lower limit, because not all of the YSOs have been found. Indeed, the poster contribution by Smith et al. in these proceedings shows the new 


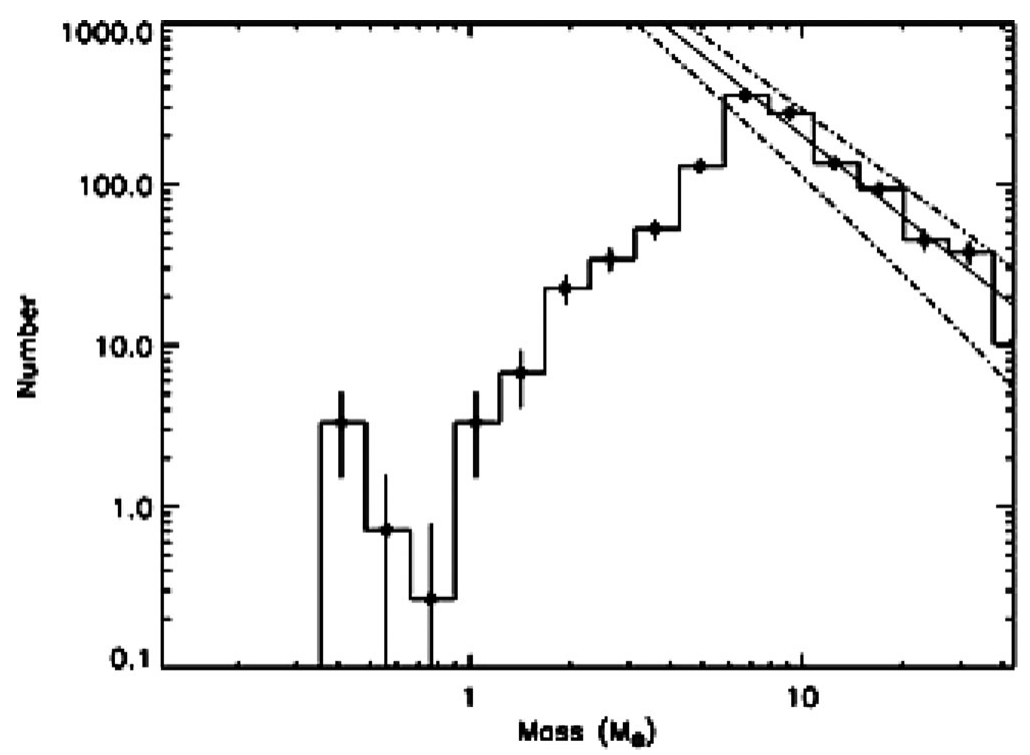

Figure 2. Histograms of stellar mass for the well-fit high-probability YSO candidates from Whitney et al. (2008). The dashed line in the left panel is the (Kroupa 2001) initial mass function.

mosaicked photometry catalogs from SAGE-LMC, and reveals 5 times more additional YSO candidates using the same color cut criteria as Whitney et al. (2008), and 30 times more additional YSO candidates if we include the fainter magnitudes in the cut for the Henize 206 and N 11 star formation regions.

\section{Mass-loss rate return from AGB stars}

The identification of the evolved star populations for the whole SAGE-LMC catalogs has been presented by Blum et al. (2006) who suggests there may be two types of oxygen-rich (O-rich) AGB stars. Srinivasan et al. (2008, also poster contribution in these proceeding), confirms these two types of O-rich AGB stars and also investigates the properties of carbon-rich (C-rich) and extreme AGB stars, in particular, the excess infrared emission in the IRAC and MIPS $24 \mu \mathrm{m}$ bands. The infrared excess measured in these stars is an excess above the expected photospheric emission in these bands, as known from scaled model atmosphere profiles. The excess is due to the dusty, molecular rich mass loss experienced by AGB stars. The larger the excess, the larger the mass-loss rate the AGB star is experiencing. Srinivasan et al. found a positive, strong correlation between the measured excess at $8 \mu \mathrm{m}$ of $\sim 40$ evolved stars for which mass-loss rates were derived from radiative transfer model fits of ISO data by van Loon et al. (1999). They made fits to these relations for the three classes of AGB stars under consideration: O-rich, C-rich and Extreme. Applying this relation to the entire sample of AGB stars, they estimate a mass-loss rate for each candidate AGB star in the sample. Figure 3 shows the cumulative mass-loss rate for the entire populations of AGB stars. The extreme AGB stars are the major contributors to the mass-loss rate, at $\sim 7 \times 10^{-3} \mathrm{M}_{\odot}$ $\mathrm{yr}^{-1}$. The C-rich AGB stars and O-rich AGB stars contribute $\sim 1 \times 10^{-3}$ and $0.7 \times 10^{-3}$ $\mathrm{M}_{\odot} \mathrm{yr}^{-1}$, respectively. The total mass-loss rate is $>8.7 \times 10^{-3} \mathrm{M}_{\odot} \mathrm{yr}^{-1}$ (see Table 1 ). We set this as a lower limit, because the AGB stars are still candidates and need further analysis, particularly at the most luminous end. Additional evolved star results based on 


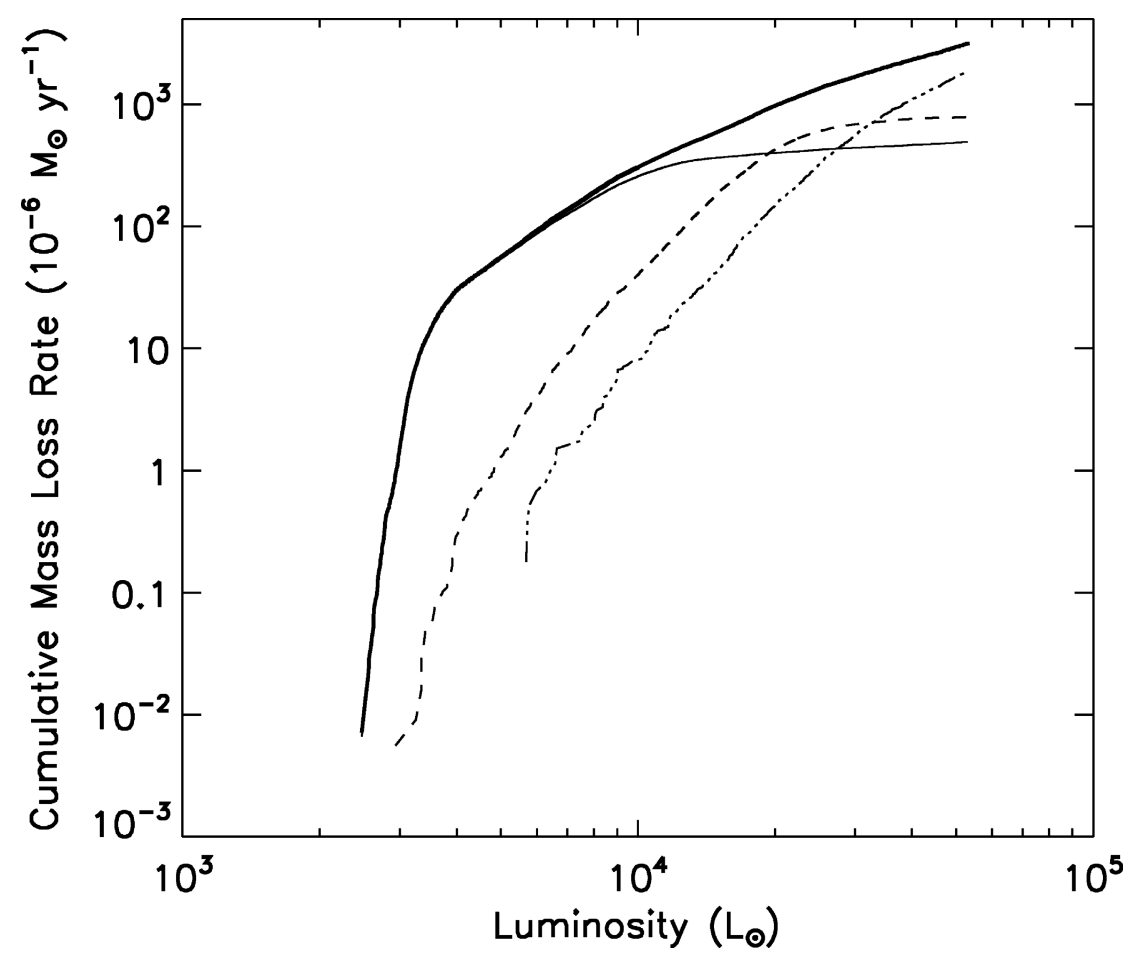

Figure 3. A preliminary cumulative mass-loss rate as a function of luminosity for the O-rich (thin solid line), C-rich (dashed line) and Extreme (dot-dashed line) AGB stars in our lists. The thick solid line is the total AGB mass-loss rate as a function of luminosity. From Srinivasan et al. (2008).

Table 1. SAGE-LMC Inventory of the Baryonic Mass Life Cycle

\begin{tabular}{lll}
\hline Item & Value & Reference \\
\hline Stellar Mass & $3 \times 10^{9} \mathrm{M}_{\odot}$ & van der Marel et al. $(2002)$ \\
ISM Mass & $10^{9} \mathrm{M}_{\odot}$ & Bernard et al. $(2008)$ \\
Star Formation Rate & $>0.1 \mathrm{M}_{\odot} \mathrm{yr}^{-1}$ & Whitney et al. $(2008)$ \\
AGB Mass Loss Return & $>8.7 \times 10^{-3} \mathrm{M}_{\odot} \mathrm{yr}^{-1}$ & Srinivasan et al. $(2008)$ \\
\hline
\end{tabular}

the SAGE-LMC data are presented in these proceedings by Vijh et al. (infrared variables discovered comparing epoch 1 and 2 data), Meixner et al. (comparison of SAGE and MACHO data on AGB stars), Srinivasan et al. (near-IR spectroscopic followup), Ueta et al. (post-AGB stars), and Cohen et al. (planetary nebulae).

\section{Inventory of Baryonic Mass Flow in the LMC}

The mass inventory of these three components, ISM, star formation and AGB mass loss, is presented in Table 1. Our new measurement of the ISM mass makes it about one third that of the stellar mass. The formation of new stars appears to be consuming the ISM at a faster rate than the AGB stars are replenishing it. However, both of these rates are preliminary and most likely lower limits to the actual rates. Moreover, they represent a current snap shot in the lifetime of the LMC and we would need to incorporate the the star formation and evolution history to fully interpret. As we work to improve this 
inventory of baryonic matter, we will add the contributions of planetary nebulae, red supergiants, luminous blue variables, supernova (remnants), and infall and outflow caused by tidal interactions with nearby gas and the SMC. The SAGE-LMC and SAGE-SMC projects will be able to provide a means to calibrate such measurements from infrared observations and act as a proving ground for procedures in making such inventories in nearby galaxies. Such calibrations will be important for missions like JWST, which will be able to extend such studies to nearby galaxies.

\section{Acknowledgement}

The SAGE Project is supported by NASA/Spitzer grant 1275598 and NASA NAG512595 .

\section{References}

Bernard, J.-P., Reach, W. T., Paradis, D., et al. 2008, AJ, 136, 919

Blum, R. D., Mould, J. R., Olsen, K. A., et al. 2006, AJ, 132, 2034

Fukui, Y., Mizuno, N., Yamaguchi, R., et al. 1999, PASJ, 51, 745

Kim, S., Staveley-Smith, L., Dopita, M. A., Sault, R. J., Freeman, K. C., Lee, Y., \& Chu, Y.-H. 2003, ApJS, 148, 473

Kroupa, P. 2001, MNRAS, 322, 231

Meixner, M., Gordon, K., Indebetouw, R., et al. 2006, AJ, 132, 2268

Srinivasan, S., Meixner, M., Leitherer, C., et al. 2008, AJ, submitted van der Marel, R. P., Alves, D. R., Hardy, E., \& Suntzeff, N. B. 2002, AJ, 124, 2639

van Loon, J. Th., Groenewegen, M. A. T., de Koter, A., Trams, N. R., Waters, L. B. F. M., Zijlstra, A. A., Whitelock, P. A., \& Loup, C. 1999, A\& $A, 351,559$

Whitney, B. A., Sewilo, M., Indebetouw, R., et al. 2008, AJ, 136, 18 